\title{
Commentary: The Influence of Proton Pump Inhibitors on the Fecal Microbiome of Infants with Gastroesophageal Reflux-A Prospective Longitudinal Interventional Study
}

\author{
Kelsea M. Drall ${ }^{1}$, Hein M. Tun ${ }^{1}$ and Anita L. Kozyrskyj ${ }^{1,2,3 *}$ \\ ${ }^{1}$ Department of Pediatrics, University of Alberta, Edmonton, AB, Canada, ${ }^{2}$ School of Public Health, University of Alberta, \\ Edmonton, AB, Canada, ${ }^{3}$ Department of Obstetrics and Gynecology, University of Alberta, Edmonton, AB, Canada
}

Keywords: proton pump inhibitors, infant gut microbiota, GERD, Clostridium difficile infection, microbiome, pre-post intervention trials, allergic diseases

\section{A Commentary on}

OPEN ACCESS

Edited by:

Yongqun $\mathrm{He}$,

University of Michigan Health System,

United States

Reviewed by:

Yuji Naito,

Kyoto Prefectural University of

Medicine, Japan

*Correspondence:

Anita Kozyrskyj

kozyrsky@ualberta.ca

Specialty section:

This article was submitted to Microbiome in Health and Disease,

a section of the journal

Frontiers in Cellular and Infection

Microbiology

Received: 24 April 2018 Accepted: 30 November 2018

Published: 14 December 2018

Citation:

Drall KM, Tun HM and Kozyrskyj AL (2018) Commentary: The Influence of

Proton Pump Inhibitors on the Fecal

Microbiome of Infants with

Gastroesophageal Reflux-A

Prospective Longitudinal Interventional

Front. Cell. Infect. Microbiol. 8:430.

doi: 10.3389/fcimb.2018.00430
The Influence of Proton Pump Inhibitors on the Fecal Microbiome of Infants with Gastroesophageal Reflux-A Prospective Longitudinal Interventional Study

by Castellani, C., Singer, G., Kashofer, K., Huber-Zeyringer, A., Flucher, C., Kaiser, M., et al. (2017). Front. Cell. Infect. Microbiol. 7:444. doi: 10.3389/fcimb.2017.00444

We are writing this commentary to discuss Castellani et al's clinical intervention trial of esomeprazole treatment for gastroesophageal reflux disease (GERD) in infants, particularly in view of the recent large-scale study by Miter et al. that reported a $\sim 1.5$-fold greater risk of allergic disease among young infants receiving proton pump inhibitors (Castellani et al., 2017; Mitre et al., 2018). Proton pump inhibitors (PPIs) are a group of medications commonly prescribed in adults and increasingly, in pediatric populations. Essentially, they increase the $\mathrm{pH}$ of the gastrointestinal tract, preventing undesirable symptoms related to gastric acidity such as vomiting and pain. In addition to allergic outcomes, other adverse side effects have also been reported with PPI treatment, notably increased risk for intestinal Clostridium difficile infection (CDI). In a meta-analysis of 23 studies, PPI use was associated with a 65\% increase in CDI risk (Janarthanan et al., 2012). Gut microbial dysbiosis with other taxa has also been reported following PPI treatment (Freedberg et al., 2015a; Shin et al., 2016; Naito et al., 2018) and is characterized by the overgrowth of Streptococcus spp., Enterococcus spp., and Escherichia spp. (Imhann et al., 2016; Takagi et al., 2018).

Castellani et al. are the first to report on the gut microbiome in relation to PPI treatment of infants with a confirmed diagnosis of GERD (Castellani et al., 2017), concluding that esomeprazole treatment of 12 study infants was not associated with significant alteration to gut microbial composition. In particular, neither diversity nor mean relative abundances of the bacteria characteristic of PPI-related dysbiosis (i.e., Streptococcus spp.) were significantly different between stool samples collected before and during PPI treatment. PPI's are also prescribed to healthy infants without confirmed GERD-almost 5\% of infants in the CHILD (Canadian Healthy Infant Longitudinal Development) cohort received a PPI before they reached the age of 3 months. Hence, we wish to further comment on the findings presented by Castellani et al. and identify issues with their interpretation.

Castellani et al. conducted a pre-post intervention design to test the efficacy and adverse effects of an $\sim 18$-week treatment course of esomeprazole, a study design that has major limitations for studying the developing infant gut microbiome. This is because microbial diversity of 


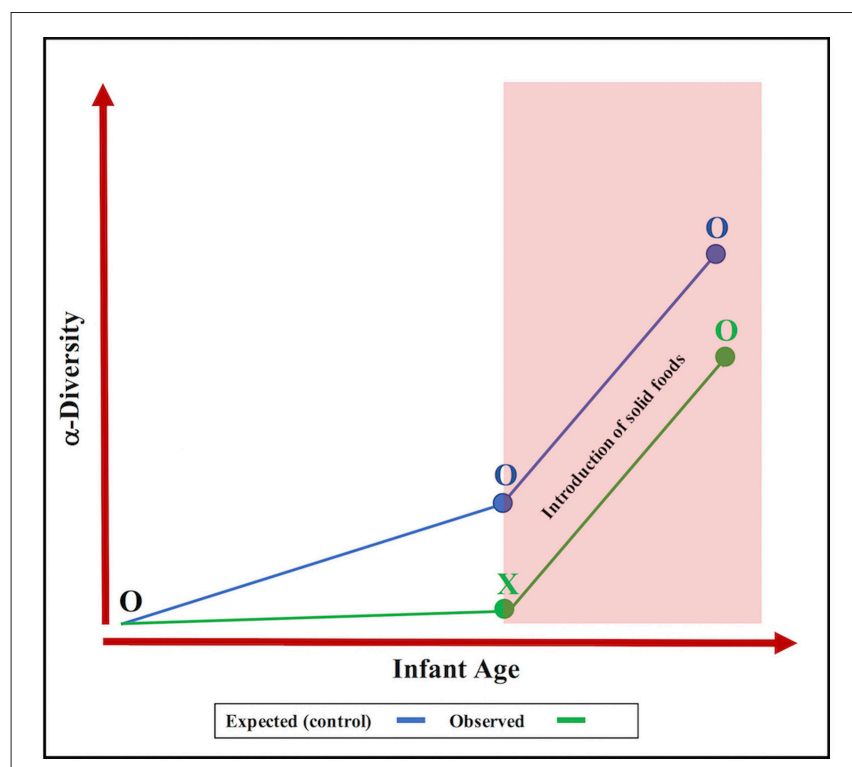

FIGURE 1 | The overlooked effect of proton pump inhibitors on the development of the infant gut microbiota. Expected progression (blue line) vs. observed progression (green line) shows that PPI intervention $(X)$ may inhibit the expansion of $\alpha$-diversity $(\mathrm{O}=$ no treatment).

the infant gut naturally increases with advancing age and in response to feeding practices (Bäckhed et al., 2015), as was shown in the Castellani paper following the discontinuation of PPI therapy. While Castellani et al. correctly identified age and feeding status as confounding factors, they failed to comment on the noticeably absent expansion of infant gut microbial diversity during the initial 4 weeks of esomeprazole treatment. We propose that this lack of significant increase to microbial diversity is a consequence of the PPI medication itself (Figure 1). Our hypothesis is supported by many studies in adults that report lower gut microbial richness and diversity with PPI use (Imhann et al., 2016; Jackson et al., 2016). Thus, a requisite addition to the pre-post study design would have been a control group of healthy untreated infants. Once matched on age, birth mode and feeding status, it is unlikely that the microbiome of a control group would be more heterogeneous than that of the infants included in the Castellani study.

Furthermore, Castellani and colleagues were hesitant to discuss trends supporting PPI-induced dysbiosis (higher

\section{REFERENCES}

Bäckhed, F., Roswall, J., Peng, Y., Feng, Q., Jia, H., Kovatcheva-Datchary, P., et al. (2015). Dynamics and stabilization of the human gut microbiome duing the first year of life. Cell Host Microbe 17, 690-703. doi: 10.1016/j.chom.2015.04.004

Castellani, C., Singer, G., Kashofer, K., Huber-Zeyringer, A., Flucher, C., Kaiser, M., et al. (2017). The influence of proton pump inhibitors on the fecal microbiome of infants with gastroesophageal reflux-A prospective longitudinal interventional study. Front. Cell Infect. Microbiol. 7:444. doi: $10.3389 /$ fcimb. 2017.00444 abundance of Streptococcus and Enterococcus species, reduced Clostridiaceae), which may have reached statistical significance with a larger sample size. These compositional changes are evident in several studies of adult PPI use (Freedberg et al., 2015b), including the ones we cited above. Recognizing that PPI-associated changes to the adult fecal microbiome may be more pronounced, consistency between infants and adults in the nature of observed compositional changes to individual microbial species are worth emphasizing. An upward trend in the abundance of Streptococcus spp. was noticeable among esomeprazole-treated infants despite a small sample size (Castellani et al., 2017). In fact, streptococci also become more abundant in the oral microbiome following esomeprazole treatment which continues to support the universal and concerning impact of these medications on the microbiota (Mishiro et al., 2018).

Finally, although the authors commented on the influence of age, they did not report the individual ages of the 12 participating infants. It appears that the study population is biased toward an older cohort ( $>6$ months of age) because 11 of 12 infants were fed solid foods by the end of the study period. Additionally, all study participants had a proven diagnosis of GERD. Meanwhile, the peak incidence of "naturally-occurring" gastroesophageal reflux symptoms occurs at $\sim 4$ months after birth due to immature esophageal musculature (Martin et al., 2002; Lightdale and Gremse, 2013). As such, we point to the possibility of "indication creep" for the PPI treatment of infant colic and spitting symptoms against a backdrop of insufficient evidence of drug efficacy (Gieruszczak-Białek et al., 2015). Expansion of PPI use may be accompanied by a real danger of disrupted development of the infant gut microbiome and subsequently, future allergic disease. In closing, we applaud Castellani and colleagues for implementing an assessment of the risk of gut microbiome dysbiosis in a trial of PPI treatment in infants. However, such a study requires the inclusion of appropriate control subjects to discern the effect of PPI use on the colonization and early development of the infant gut microbiome.

\section{AUTHOR CONTRIBUTIONS}

KMD drafted and revised the manuscript. ALK initiated and revised the manuscript. HMT provided feedback and suggestions. All authors approved the final version of the manuscript for submission.

Freedberg, D. E., Lamousé-Smith, E. S., Lightdale, J. R., Jin, Z., Yang, Y. X. and Abrams, J. A. (2015a). Use of acid suppression medication is associated with risk for $C$. Difficile Infection in infants and children: a population-based study. Clin. Infect. Dis. 61, 912-917. doi: 10.1093/cid/civ432

Freedberg, D. E., Toussaint, N. C., Chen, S. P., Ratner, A. J., Whittier, S., Wang, T. C., et al. (2015b). Proton pump inhibitors alter specific taxa in the human gastrointestinal microbiome: a crossover trial. Gastroenterology 149, 883-85. doi: 10.1053/j.gastro.2015.06.043

Gieruszczak-Białek, D., Konarska, Z., Skórka, A., Vandenplas, Y., and Szajewska, H. (2015). No effect of proton pump inhibitors on crying and irritability 
in infants: systematic review of randomized controlled trials. J Pediatr. 166, 767-770. doi: 10.1016/j.jpeds.2014.11.030

Imhann, F., Bonder, M. J.,Vich Vila, A., Fu, J., Mujagic, Z., Vork, L., et al. (2016). Proton pump inhibitors affect the gut microbiome. Gut 65, 740-748. doi: 10.1136/gutjnl-2015-310376

Jackson, M. A., Goodrich, J. K., Maxan, M. E., Freedberg, D. E., Abrams, J. A., Poole, A. C., et al. (2016). Proton pump inhibitors alter the composition of the gut Microb. Gut. 65, 749-756. doi: 10.1136/gutjnl-2015-310861

Janarthanan, S., Ditah, I., Adler, D. G., and Ehrinpreis, M. N. (2012). Clostridium difficile-associated diarrhea and proton pump inhibitor therapy: a meta-analysis. Am. J. Gastroenterol. 107, 1001-1010. doi: 10.1038/ajg. 2012.179

Lightdale, J. R., and Gremse, D. A. (2013). Gastroesophageal reflux: Management guidance for the pediatrician. Pediatrics 131, 1684-1695. doi: 10.1542/peds.2013-0421

Martin, A. J., Pratt, N., Kennedy, J. D., Ryan, P., Ruffin, R. E., Miles, H., et al. (2002). Natural history and familial relationships of infant spilling to 9 years of age. Pediatrics 109, 1061-1067. doi: 10.1542/peds.109.6.1061

Mishiro, T., Oka, K., Kuroki, Y., Takahashi, M., Tatsumi, K., Saitoh, T., et al. (2018). Oral Microbiome alterations of healthy volunteers with proton pump inhibitor. J. Gastroenterol. Hepatol. 33, 1059-1066. doi: 10.1111/jgh. 14040

Mitre, E., Susi, A. Kropp, L.E., Schwartz, D.J., Gorman, G.H., and Nylund, C.M. (2018). Associations between use of acid-suppressive medications and antibiotics during infancy and allergic diseases in early childhood. JAMA Pediatr. 172:e180315. doi: 10.1001/jamapediatrics.2018.0315

Naito, Y., Kashiwagi, K., Takagi, T., Andoh, A. and Inoue, R. (2018). Intestinal dysbiosis secondary to proton-pump inhibitor use. Digestion 8566, 195-204. doi: $10.1159 / 000481813$

Shin, C. M., Kim, N., Kim, Y. S., Nam, R. H., Park, J. H., Lee, D. H., et al. (2016). Impact of long-term proton pump inhibitor therapy on gut microbiota in F344 rats: a pilot study. Gut Liver 10, 896-901. doi: 10.5009/ gnl15529

Takagi, T., Naito, Y., Inoue, R., Kashiwagi, S., Uchiyama, K., Mizushima, K., et al. (2018). The Influence of long-term use of proton pump inhibitors on the gut microbiota: an age-sex-matched case-control study. J. Biochem. Nutr. 62, 100-105. doi: $10.3164 /$ jcbn. $17-78$

Conflict of Interest Statement: The authors declare that the research was conducted in the absence of any commercial or financial relationships that could be construed as a potential conflict of interest.

Copyright $\odot 2018$ Drall, Tun and Kozyrskyj. This is an open-access article distributed under the terms of the Creative Commons Attribution License (CC BY). The use, distribution or reproduction in other forums is permitted, provided the original author(s) and the copyright owner(s) are credited and that the original publication in this journal is cited, in accordance with accepted academic practice. No use, distribution or reproduction is permitted which does not comply with these terms. 